\title{
Pacific
}

Journal of

Mathematics

KNOTTING TRIVIAL KNOTS AND RESULTING KNOT TYPES

KIMIHIKO MOTEGI

Volume $161 \quad$ No. 2

December 1993 


\section{KNOTTING TRIVIAL KNOTS AND RESULTING KNOT TYPES}

\section{KIMIHIKO MOTEGI}

Let $(V, K)$ be a pattern (i.e. $V$ is a standardly embedded solid torus in oriented $S^{3}$ and $K$ is a knot in $V$ ) and $f$ an orientation preserving emdedding from $V$ into $S^{3}$ such that $f(V)$ is knotted.

In this paper answers to the following questions will be given depending upon whether the winding number of $K_{2}$ in $V$ is zero or not.

(1) Suppose that $K_{1}$ is unknotted and $K_{2}$ is knotted in $S^{3}$. Can $f\left(K_{1}\right)$ be ambient isotopic to $f\left(K_{2}\right)$ in $S^{3}$ for some embedding $f$ : $V \hookrightarrow S^{3}$ ?

(2) Suppose that $K_{1}$ and $K_{2}$ are both unknotted in $S^{3}$. How are $\left(V, K_{1}\right)$ and $\left(V, K_{2}\right)$ related if $f\left(K_{1}\right)$ is ambient isotopic to $f\left(K_{2}\right)$ in $S^{3}$ for some embedding $f: V \hookrightarrow S^{3}$ ?

1. Introduction. Let $K$ be a knot in $S^{3}$, which is contained in a standardly embedded solid torus $V\left(\subset S^{3}\right)$. Assume that $K$ is not contained in a 3-ball in $V$. Let $f$ be an orientation preserving embedding from $V$ into $S^{3}$ such that $f(C)$ is knotted in $S^{3}$, here $C$ denotes a core of $V$. Then we get a new knot $f(K)$ in $S^{3}$ called a satellite knot with a companion knot $f(C)$. The knot $K$ is called a preimage knot and we call the pair $(V, K)$ a pattern (see Figure 1 on the next page).

Throughout this paper for an embedding $f$ from $V$ into $S^{3}$, we assume that it is orientation preserving and $f(C)$ is knotted in $S^{3}$.

We concern ourselves with the following questions.

(1) Suppose that $K_{1}$ is unknotted and $K_{2}$ is knotted in $S^{3}$. Can $f\left(K_{1}\right)$ be ambient isotopic to $f\left(K_{2}\right)$ in $S^{3}$ for some embedding $f$ : $V \hookrightarrow S^{3}$ ?

(2) Suppose that $K_{1}$ and $K_{2}$ are both unknotted in $S^{3}$. How are $\left(V, K_{1}\right)$ and $\left(V, K_{2}\right)$ related if $f\left(K_{1}\right)$ is ambient isotopic to $f\left(K_{2}\right)$ in $S^{3}$ for some embedding $f: V \hookrightarrow S^{3}$ ?

For two knots $K_{1}$ and $K_{2}$, we write $K_{1} \cong K_{2}$ provided that there exists an orientation preserving self-homeomorphism of $S^{3}$ carrying $K_{1}$ to $K_{2}$ (or equivalently, $K_{1}$ and $K_{2}$ are ambient isotopic in $S^{3}$ ). For two patterns $\left(V, K_{1}\right)$ and $\left(V, K_{2}\right)$, if there exists an orientation preserving self-homeomorphism $h$ of $V$ sending longitude to 

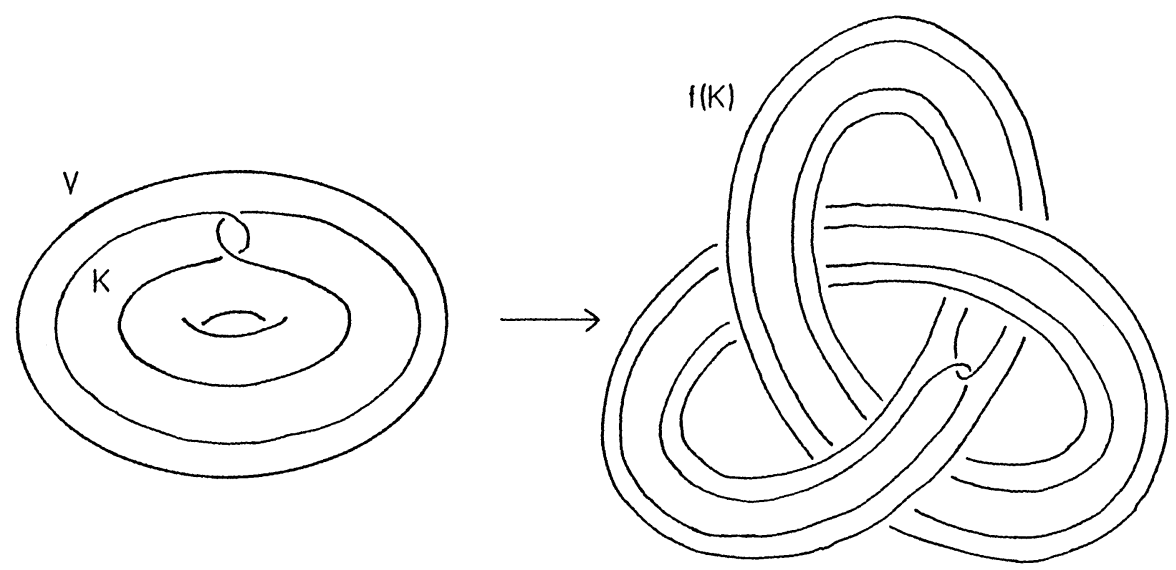

Figure 1

Ilongitude which satisfies $h\left(K_{1}\right)=K_{2}$, then we write $\left(V, K_{1}\right) \sim$ $\left(V, K_{2}\right)$. In addition if the homeomorphism $h$ sends longitude to longitude, then we write $\left(V, K_{1}\right) \cong\left(V, K_{2}\right)$. It is known that $\left(V, K_{1}\right)$ $\cong\left(V, K_{2}\right)$ if and only if $K_{1}$ and $K_{2}$ are ambient isotopic in $V$. Throughout this paper longitude means preferred longitude.

The wrapping number of $K$ in $V$-the minimal geometric intersection number of $K$ with a meridian disk in $V$-is denoted by $\operatorname{wrap}_{V}(K)$, and the winding number of $K$ in $V$-the algebraic intersection number of $K$ with a meridian disk in $V$-is denoted by wind $_{V}(K)$. (We may assume wind $_{V}(K) \geq 0$ by considering an appropriate orientation of $K$.)

Now our main result is stated as follows.

THEOREM 1.1. Let $\left(V, K_{i}\right)(i=1,2)$ be a pattern. Suppose that $K_{1}$ is unknotted in $S^{3}$ and wind $_{V}\left(K_{2}\right) \neq 0$. If $f\left(K_{1}\right) \cong f\left(K_{2}\right)$ in $S^{3}$ for some embedding $f$ from $V$ into $S^{3}$, then $\left(V, K_{1}\right) \sim\left(V, K_{2}\right)$ holds.

REMARK 1.2. (1) In this theorem the condition wind $_{V}\left(K_{2}\right) \neq 0$ is essential. The example below (Figure 2 ) demonstrates the necessity of such a condition.

In this example $K_{1}$ is unknotted in $S^{3}$ and $K_{2}$ is knotted (and hence $\left.\left(V, K_{1}\right) \not\left(V, K_{2}\right)\right)$, but $\operatorname{wind}_{V}\left(K_{2}\right)=0$. From them, we can obtain the same knot $f\left(K_{1}\right) \cong f\left(K_{2}\right)$.

The modification to recognize that $f\left(K_{1}\right) \cong f\left(K_{2}\right)$ is given by Figure 3. 

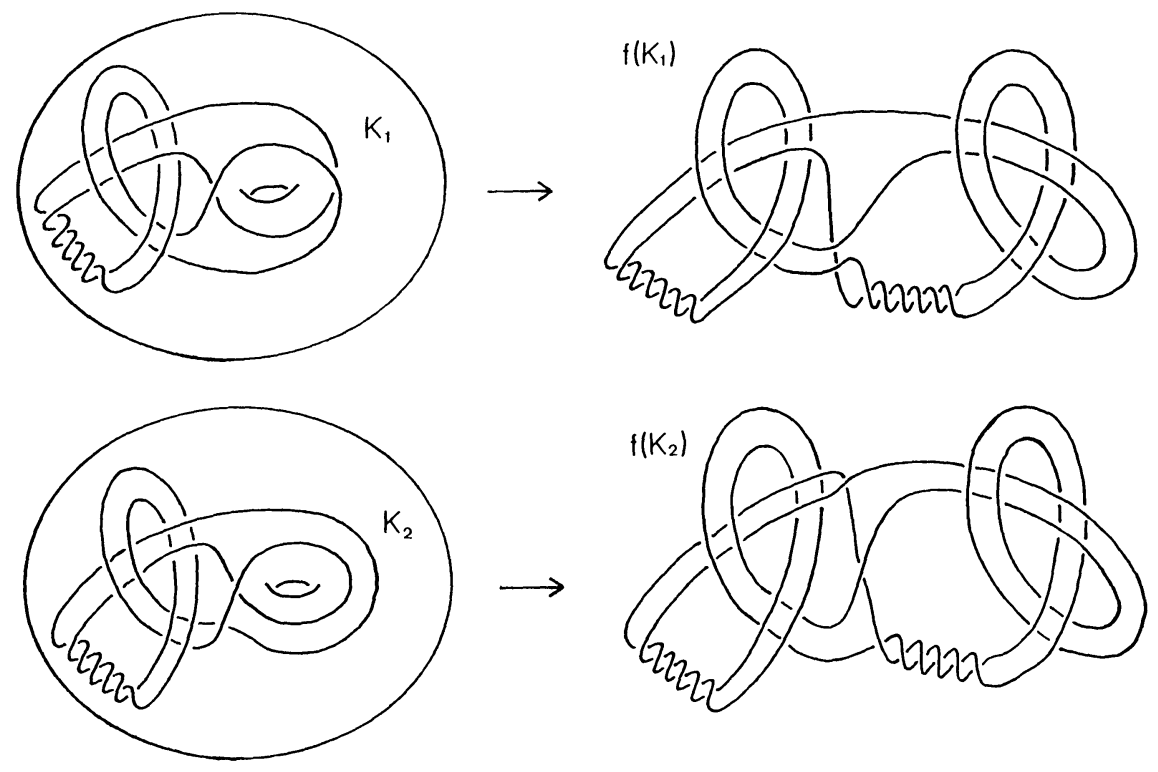

FIGURE 2

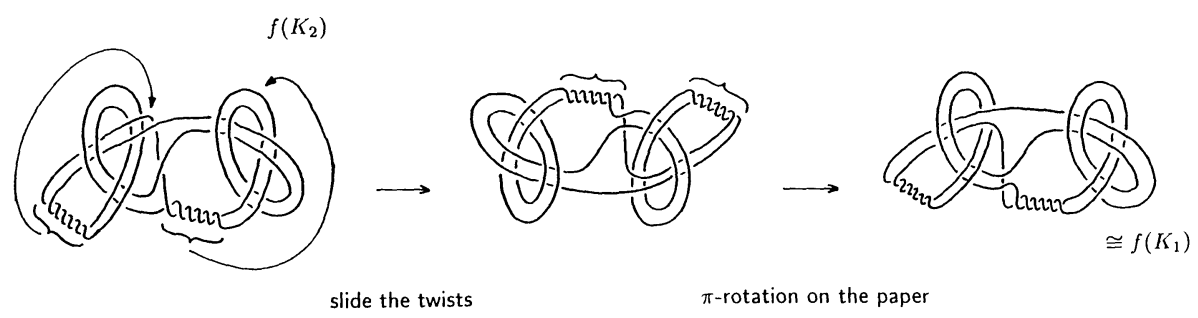

FIGURE 3
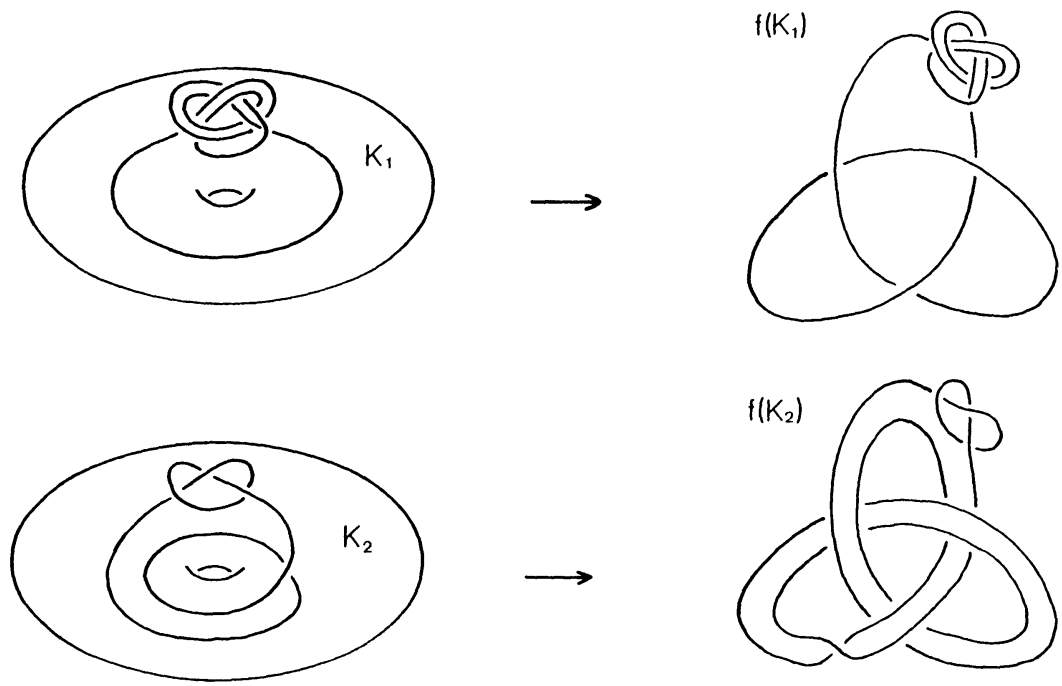

Figure 4 
(2) If $K_{1}$ is knotted, even when wind $_{V}\left(K_{2}\right) \neq 0$, it is easy to construct the example such that $\left(V, K_{1}\right) \not\left(V, K_{2}\right)$ but $f\left(K_{1}\right) \cong f\left(K_{2}\right)$ in $S^{3}$ (see Figure 4).

As consequences of the Theorem 1.1, we have Corollary 2.6 and Theorem 3.1. By these results together with Remark 1.2 and Theorem 3.3, we can answer the above questions depending upon whether wind $_{V}\left(K_{2}\right)=0$ or not.

Throughout this paper $N(X), \partial X$ and int $X$ denote the tubular neighborhood of $X$, the boundary of $X$ and the interior of $X$ respectively.

2. Isotopy between satellite knots and equivalence of patterns. To prove Theorem 1.1 we prepare some lemmas and give a necessary condition for a pattern $(V, K)$ so that $K$ is unknotted in $S^{3}$.

The next lemma is well known and we omit the proof here (see [7]).

LEMMA 2.1. Let $W$ be a knotted solid torus in $S^{3}$ and $K$ a knot in $W$ with $\operatorname{wrap}_{W}(K) \neq 0$. Then $K$ is knotted in $S^{3}$.

Consider a nontrivial knot exterior $E$ (i.e. $E$ is homeomorphic to $S^{3}$ - int $N(k)$ for some nontrivial knot $k$ in $S^{3}$ ) embedded in $V$. Since $\partial E$ is compressible in $V$ - int $N(K)$, otherwise $V=$ $(V$ - int $E) \cup E$ has an incompressible torus $\partial E$, there exists an embedded disk $D$ in $V$ - int $E$ such that $\partial D$ is essential in $\partial(V-\operatorname{int} E)=\partial E$. Thus $D$ is a meridian disk for the solid torus $W=S^{3}-$ int $E$ and is contained in $V$. We call the disk $D$ a meridian disk for $E$ in $V$. The following lemma is a straightforward consequence of Lemma 2.1.

LEMMA 2.2. Let $(V, K)$ be a pattern such that $K$ is unknotted in $S^{3}$ and $E$ a nontrivial knot exterior embedded in $V-K$. Then the algebraic intersection number of $K$ and a meridian disk for $E$ in $V$ is zero (see Figure 5).

Now we shall prove Theorem 1.1.

Proof of Theorem 1.1. Suppose that $f\left(K_{1}\right) \cong f\left(K_{2}\right)$ in $S^{3}$ for two patterns $\left(V, K_{1}\right)$ and $\left(V, K_{2}\right)$. Then there is an orientation preserving homeomorphism $h$ of $S^{3}$ carrying $f\left(K_{1}\right)$ to $f\left(K_{2}\right)$. It suffices to show that by an isotopy of $S^{3}$ which leaves $f\left(K_{2}\right)=h\left(f\left(K_{1}\right)\right)$ fixed, we can modify the homeomorphism $h$ so that $h\left(S^{3}-\right.$ int $\left.f(V)\right)=S^{3}-$ int $f(V)$. To do this we need the next lemma, which was proved by $\mathrm{H}$. Schubert in [8], but since we rely heavily on this theorem, we give a proof here using torus decompositions. 


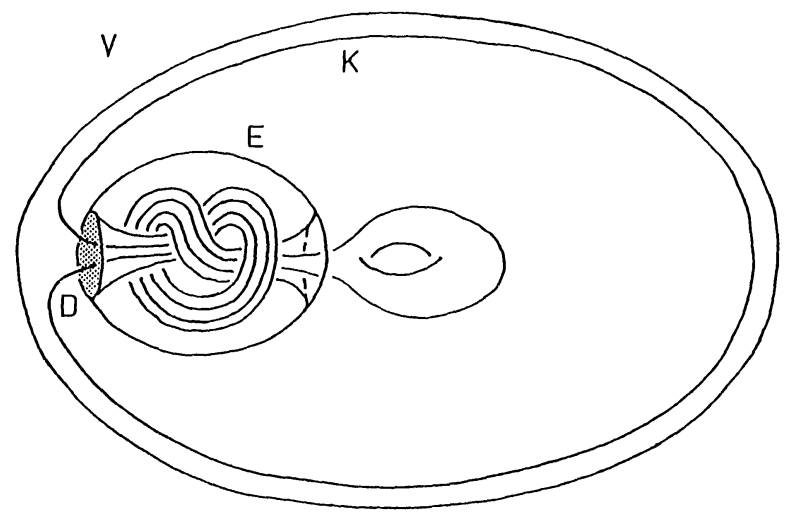

FIGURE 5

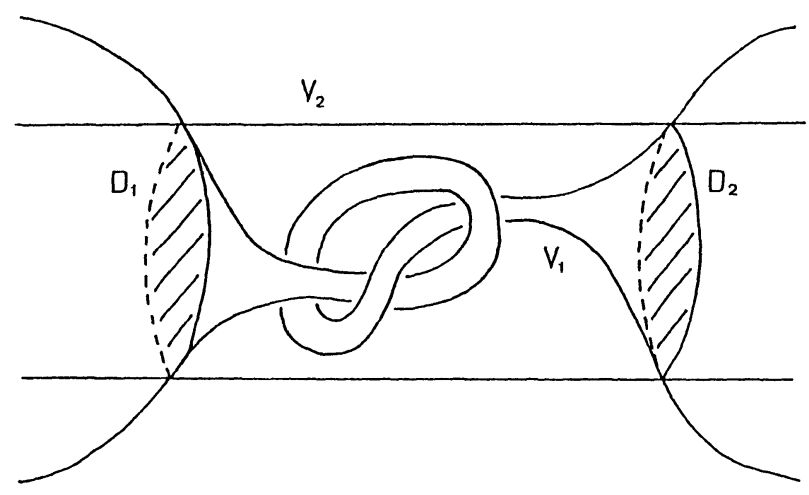

FIGURE 6

LEMMA 2.3 ([8]). Let $V_{i}(i=1,2)$ be a knotted solid torus in $S^{3}$ which contains a knot $K$ with $\operatorname{wrap}_{V_{i}}(K) \neq 0$. Then by an ambient isotopy of $S^{3}$ which leaves $K$ fixed, $V_{2}$ can be deformed so that one of the following holds.

(1) $\partial V_{1} \cap \partial V_{2}=\varnothing$

(2) there exist meridian disks $D_{1}$ and $D_{2}$ of both $V_{1}$ and $V_{2}$ such that the closure of one component of $V_{1}-\bigcup_{i=1}^{2} D_{i}$ is a knotted 3-ball in the closure of some component of $V_{2}-\bigcup_{i=1}^{2} D_{i}$ (see Figure 6).

Proof of Lemma 2.3. If $V_{i}$ int $N(K)$ is homeomorphic to $S^{1} \times$ $S^{1} \times I$ for $i=1$ or 2 , then $K$ is a core of $V_{i}$ and we can deform $V_{2}$ so that (1) in Lemma 2.3 holds. Now we assume that $V_{i}-\operatorname{int} N(K)$ is not homeomorphic to $S^{1} \times S^{1} \times I$ for $i=1,2$. Consider the torus decomposition of $S^{3}-$ int $V_{i}$ and $V_{i}$ - int $N(K)(i=1,2)$ in the sense of Jaco-Shalen [4] and Johannson [5]. Then each piece is Seifert fibred or admits a complete hyperbolic structure of finite volume in its interior by Thurston's uniformization theorem [6]. Moreover, by 
Theorem VI 3.4 in [4], the Seifert part is one of torus knot spaces, cable spaces and composing spaces. Let $M_{i}$ be the piece in $S^{3}$ int $V_{i}$ which contains $\partial V_{i}\left(=\partial\left(S^{3}-\right.\right.$ int $\left.\left.V_{i}\right)\right)$ and $N_{i}$ the piece in $V_{i-}$ int $N(K)$ which contains $\partial V_{i}$. We divide into two cases depending upon whether one of $\partial V_{i}(i=1,2)$ belongs to the minimal family of tori $J_{i}$ defining a torus decomposition of $S^{3}-\operatorname{int} N(K)=\left(S^{3}-\right.$ int $\left.V_{i}\right) \cup\left(V_{i}-\right.$ int $\left.N(K)\right)$ or not.

Case (A). At least one of $\partial V_{i}(i=1,2)$ belongs to $J_{i}(i=1,2)$.

In this case, by the uniqueness of the torus decomposition we can deform $\partial V_{2}$ so that $\partial V_{1} \cap \partial V_{2}=\varnothing$ or $\partial V_{1}=\partial V_{2}$. If $\partial V_{1}=\partial V_{2}$, then isotoping $\partial V_{2}$ slightly off $\partial V_{1}$ in the normal direction so that $\partial V_{1} \cap \partial V_{2}=\varnothing$. Thus (1) in Lemma 2.3 does hold.

Case (B). $\partial V_{i}(i=1,2)$ does not belong to $J_{i}$.

Then it turns out that $M_{i} \cup N_{i}$ is a composing space in $S^{3}-$ int $N(K)$ $=\left(S^{3}-\right.$ int $\left.V_{i}\right) \cup\left(V_{i}-\right.$ int $\left.N(K)\right)$. By the uniqueness of the torus decomposition, we can isotope $M_{2} \cup N_{2}$ so that $M_{2} \cup N_{2}$ is one of decomposing pieces defined by $J_{1}$. (Notationally we do not distinguish the original $M_{2} \cup N_{2}$ and isotoped $M_{2} \cup N_{2}$.) If $M_{2} \cup N_{2} \neq M_{1} \cup N_{1}$, then (1) in Lemma 2.3 holds. Now suppose that $M_{2} \cup N_{2}=M_{1} \cup N_{1}$. We note that $\partial V_{1}$ is a saturated torus (i.e. a union of fibres) in the composing space $M_{1} \cup N_{1}$. Since a Seifert fibration of the composing space $M_{1} \cup N_{1}$ is unique up to isotopy, we may modify $\partial V_{2}$ so that it is also saturated. Consider the orbit manifold of $M_{1} \cup N_{1}$, which is a disk with holes. The image of $\partial V_{i}$ in this orbit manifold is an essential circle $C_{i}$. If we can modify $C_{2}$ so that $C_{1} \cap C_{2}=\varnothing$, then we can also modify $\partial V_{2}$ so that $\partial V_{1} \cap \partial V_{2}=\varnothing$ and (1) in Lemma 2.3 holds. Assume we can not isotope $C_{2}$ so that $C_{1} \cap C_{2}=\varnothing$. Then isotope $C_{2}$ so that the number of points of $C_{1} \cap C_{2}$ is minimal. Let $T$ be the component of $\partial\left(M_{1} \cup N_{1}\right)$ separating $\partial N(K)$ and $M_{1} \cup N_{1}$. In $S^{3}, T$ bounds a solid torus $W$ containing $K$, whose meridian coincides with the regular fibre of $M_{1} \cup N_{1}$. In the orbit manifold we can find arcs $A_{1}$ and $A_{2}$ joining a point in $C_{1} \cap C_{2}$ and the boundary circle $C$ which is the image of $T$ (see Figure 7 (1)).

These arcs $A_{1}$ and $A_{2}$ are corresponding to saturated annuli $\tilde{A}_{1}$ and $\widetilde{A}_{2}$. From $\widetilde{A}_{j}$ and meridian disk $\widetilde{D}_{j}$ of $W$, we can construct a meridian disk $\widetilde{A}_{j} \cup \widetilde{D}_{j}$ of both $V_{1}$ and $V_{2}$. Finally consider the boundary circle $C^{\prime}$ of the orbit manifold depicted in Figure 7 (1), which corresponds to the torus boundary $T^{\prime}$. Since $T^{\prime}$ bounds a 


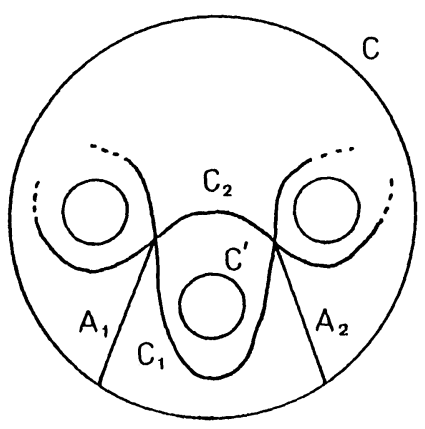

(1)

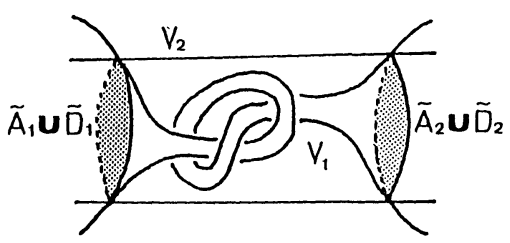

(2)

FIGURE 7

nontrivial knot exterior in $V_{2}-$ int $V_{1}$ (see Figure 7 (2)), we get just a situation for (2) in Lemma 2.3, and this completes the proof.

Let us study the relationship between two solid tori $h(f(V))$ and $f(V)$ using Lemma 2.3. By an ambient isotopy of $S^{3}$ which leaves $f\left(K_{2}\right)=h\left(f\left(K_{1}\right)\right)$ fixed we can deform $h(f(V))$ into the position such that either $\partial h(f(V)) \cap \partial f(V)=\varnothing$ or there exist meridian disks $D_{1}$ and $D_{2}$ of both $f(V)$ and $h(f(V))$ such that one component of the closure of $f(V)-\bigcup_{i=1}^{2} D_{i}$ is a knotted 3-ball in some component of the closure of $h(f(V))-\bigcup_{i=1}^{2} D_{i}$.

LEMMA 2.4. We can deform $h(f(V))$ into the position such that $\partial h(f(V)) \cap \partial f(V)=\varnothing$.

Proof of Lemma 2.4. If not, the second situation in the above occurs. Then we get a solid torus $W^{\prime}\left(\supset h\left(f\left(K_{1}\right)\right)=f\left(K_{2}\right)\right)$ in int $h(f(V))$, whose core $C_{W^{\prime}}$ satisfies $\operatorname{wrap}_{h(f(V))}\left(C_{W^{\prime}}\right)=1$ and is not a core of $h(f(V))$. It follows that the solid torus $V$ also contains a knotted solid torus $W\left(\supset K_{1}\right)$ in its interior such that $\operatorname{wrap}_{V}\left(C_{W}\right)=1$ for the core $C_{W}$ of $W$. Since $\operatorname{wrap}_{W}\left(K_{1}\right) \neq 0, K_{1}$ cannot be unknotted in $S^{3}$ by Lemma 2.1 and this contradicts the assumption. Hence we can deform $h(f(V))$ so that $\partial h(f(V)) \cap \partial f(V)=\varnothing$.

Now we have following three possibilities.

(1) $h\left(S^{3}-\right.$ int $\left.f(V)\right) \subset$ int $\left(S^{3}-\right.$ int $\left.f(V)\right)$

(2) int $h\left(S^{3}-\right.$ int $\left.f(V)\right) \supset S^{3}-$ int $f(V)$

(3) $h\left(S^{3}-\right.$ int $\left.f(V)\right) \subset$ int $f(V)$

In (1) (or (2), resp.), assume $\left(S^{3}-i\right.$ int $\left.f(V)\right)-\operatorname{int}\left(h\left(S^{3}-\right.\right.$ int $\left.\left.f(V)\right)\right)$ (or $h\left(S^{3}-\right.$ int $\left.f(V)\right)-\operatorname{int}\left(S^{3}-\right.$ int $\left.f(V)\right)$, resp.) is homeomorphic 
to $S^{1} \times S^{1} \times I$. Then by an isotopy which leaves $f\left(K_{2}\right)$ fixed, we may modify $h$ so that $h\left(S^{3}-\right.$ int $\left.f(V)\right)=S^{3}-$ int $f(V)$ and also $h(f(V))=f(V)$ with $h\left(f\left(K_{1}\right)\right)=f\left(K_{2}\right)$. For homological reasons, $h$ (longitude $)= \pm$ longitude. Hence $f^{-1} \circ h \circ f: V \rightarrow V$ is an orientation preserving homeomorphism carrying $K_{1}$ to $K_{2}$ and longitude to \pm longitude. So we get $\left(V, K_{1}\right) \sim\left(V, K_{2}\right)$.

Let us consider the case where $h\left(S^{3}-\right.$ int $\left.f(V)\right) \subset \operatorname{int}\left(S^{3}-\right.$ int $\left.f(V)\right)$ and $\left(S^{3}-\right.$ int $\left.f(V)\right)-\operatorname{int}\left(h\left(S^{3}-\right.\right.$ int $\left.\left.f(V)\right)\right)$ is not homeomorphic to $S^{1} \times S^{1} \times I$. Then using the homeomorphism $\left.h\right|_{S^{3}-\operatorname{int} f(V)}$ from $S^{3}$ int $f(V)$ to $h\left(S^{3}-\right.$ int $\left.f(V)\right)$, we get mutually nonparallel incompressible tori $\left\{h^{n}(\partial f(V))\right\}$ in $S^{3}-$ int $f(V)$ for any positive integer $n$. This contradicts Haken's finiteness theorem. The similar argument can be applied in the case where int $h\left(S^{3}-\right.$ int $\left.f(V)\right) \supset S^{3}-$ int $f(V)$ and $h\left(S^{3}-\right.$ int $\left.f(V)\right)-\operatorname{int}\left(S^{3}-\right.$ int $\left.f(V)\right)$ is not homeomorphic to $S^{1} \times S^{1} \times I$, and again we get a contradiction.

Let us consider the case (3). The assumption implies that the nontrivial knot exterior $E=h^{-1}\left(S^{3}-\right.$ int $\left.f(V)\right)$ is contained in int $f(V)$. Since $\operatorname{wind}_{V}\left(K_{2}\right) \neq 0$, we have wind $f(V)\left(f\left(K_{2}\right)\right) \neq 0$ and so we have wind $_{h^{-1}(f(V))}\left(h^{-1}\left(f\left(K_{2}\right)\right)\right) \neq 0$. It follows that $\operatorname{wind}_{\left(S^{3}-\operatorname{int} E\right)}\left(f\left(K_{1}\right)\right)$ is also not zero. On the other hand since $K_{1}$ is unknotted in $S^{3}$, by Lemma 2.2 , the algebraic intersection number of $K_{1}$ and a meridian disk for the nontrivial knot exterior $f^{-1}(E)$ in $V$ must be zero. Hence we get wind ${ }_{\left(S^{3}-\text { int } E\right)}\left(f\left(K_{1}\right)\right)=0$. This is a contradiction.

In Theorem 1.1 , if $f(C)$ is a noninvertible knot, where $C$ is a core of $V$, then more precisely we have the following.

THEOREM 2.5. Let $\left(V, K_{i}\right)$ be a pattern. Suppose that $K_{1}$ is unknotted in $S^{3}$, and wind $_{V}\left(K_{2}\right) \neq 0$. If $f\left(K_{1}\right) \cong f\left(K_{2}\right)$ in $S^{3}$ for some embedding $f$ from $V$ into $S^{3}$ such that $f(C)$ is noninvertible, then $\left(V, K_{1}\right) \cong\left(V, K_{2}\right)$, that is $K_{1}$ and $K_{2}$ are ambient isotopic in $V$.

Proof. In the proof of Theorem 1.1, we have an orientation preserving homeomorphism $h$ of $S^{3}$ satisfying $h(f(V))=f(V)$ and $h\left(f\left(K_{1}\right)\right)=f\left(K_{2}\right)$. For homological reasons, $h$ (longitude) $\equiv$ tlongitude. In addition since $f(C)$ is noninvertible, we get $h($ longitude $)=$ longitude (see 3.19. Proposition in [1]). It follows that $f^{-1} \circ h \circ f: V \rightarrow V$ is an orientation preserving homeomorphism carrying $K_{1}$ to $K_{2}$ and longitude to longitude. Thus we conclude $\left(V, K_{1}\right) \cong\left(V, K_{2}\right)$. 
As an application of Theorem 1.1, we have the following corollary.

COROllary 2.6. Let $\left(V, K_{i}\right)$ be a pattern. Suppose that $K_{1}$ is unknotted and $K_{2}$ is knotted in $S^{3}$ and $\operatorname{wind}_{V}\left(K_{2}\right) \neq 0$. Then for any embedding $f$ from $V$ into $S^{3}, f\left(K_{1}\right) \neq f\left(K_{2}\right)$ in $S^{3}$.

Proof. If $f\left(K_{1}\right) \cong f\left(K_{2}\right)$ for some embedding $f$ from $V$ into $S^{3}$, then $\left(V, K_{1}\right) \sim\left(V, K_{2}\right)$ must hold by Theorem 1.1. Extending the orientation preserving homeomorphism $h$ of $V$ to that of $S^{3}$, we get $K_{1} \cong K_{2}$. This is a contradiction.

Concluding this section, we give the following proposition which is an implicit corollary of Soma's sum formula for the Gromov invariants [10]. We denote the Gromov invariant of $X$ by $\|X\|$.

Proposition 2.7. Let $\left(V, K_{i}\right)(i=1,2)$ be a pattern such that $\left\|V-\operatorname{int} N\left(K_{1}\right)\right\| \neq\left\|V-\operatorname{int} N\left(K_{2}\right)\right\|$. Then $f\left(K_{1}\right) \not f\left(K_{2}\right)$ for any embedding $f$ from $V$ into $S^{3}$.

So we see that, with no conditions on $K_{1}$ and $K_{2}$, if $f\left(K_{1}\right) \cong f\left(K_{2}\right)$ then the Gromov invariants of their complements in $V$ are the same.

3. Classification of satellite knots constructed from trivial knots. As a special case of Theorem 1.1, we have

THEOREM 3.1. Let $\left(V, K_{i}\right)$ be a pattern and $K_{i}$ a trivial knot in $S^{3}(i=1,2)$. Suppose that $\operatorname{wind}_{V}\left(K_{1}\right) \neq 0$ or $\operatorname{wind}_{V}\left(K_{2}\right) \neq 0$. If $f\left(K_{1}\right) \cong f\left(K_{2}\right)$ in $S^{3}$ for some embedding $f$ from $V$ into $S^{3}$, then $\left(V, K_{1}\right) \sim\left(V, K_{2}\right)$ holds.

The winding numbers and the wrapping numbers of knots in a solid torus are elementary invariants for them. Particularly for a faithful (i.e. sending longitude to longitude) embedding $f: V \hookrightarrow S^{3}$, winding number of $K$ in $V$ has an important role for Alexander polynomial of $f(K)$, as is shown by Seifert's formula [9] ([1]). However if $K$ is unknotted and $f(C)$ has a trivial Alexander polynomial, then $f(K)$ has also a trivial one independent of $\operatorname{wind}_{V}(K)$. Moreover when $K_{1} \cong K_{2}$ and $\operatorname{wind}_{V}\left(K_{1}\right)=\operatorname{wind}_{V}\left(K_{2}\right), f\left(K_{1}\right)$ and $f\left(K_{2}\right)$ have the same Alexander polynomial.

As a consequence of Theorem 3.1, we have the following result for satellite knots constructed from trivial knots. 
Corollary 3.2. Suppose $K_{i}$ is a trivial knot contained in a standardly embedded solid torus $V$ in $S^{3}(i=1,2)$.

(1) If wind $_{V}\left(K_{1}\right) \neq$ wind $_{V}\left(K_{2}\right)$, then $f\left(K_{1}\right) \not f\left(K_{2}\right)$ in $S^{3}$ for any embedding $f$ from $V$ into $S^{3}$.

(2) When $\operatorname{wind}_{V}\left(K_{1}\right)=\operatorname{wind}_{V}\left(K_{2}\right) \neq 0$, if $\operatorname{wrap}_{V}\left(K_{1}\right) \neq \operatorname{wrap}_{V}\left(K_{2}\right)$, then $f\left(K_{1}\right) \not f\left(K_{2}\right)$ in $S^{3}$ for any embedding $f$ from $V$ into $S^{3}$.

In the case $\operatorname{wind}_{V}\left(K_{1}\right)=$ wind $_{V}\left(K_{2}\right)=0$, we have

THEOREM 3.3. For any faithful embedding $f$ from $V$ into $S^{3}$ (i.e. $f$ sends a longitude of $V$ to a longitude of $f(V)$ ), there exist patterns $\left(V, K_{1}\right)$ and $\left(V, K_{2}\right)$ such that both $K_{1}$ and $K_{2}$ are unknotted in $S^{3}$, which satisfy the following properties:

(1) $\operatorname{wind}_{V}\left(K_{1}\right)=$ wind $_{V}\left(K_{2}\right)=0$ and $\left(V, K_{1}\right) \nsim\left(V, K_{2}\right)$.

(2) $f\left(K_{1}\right) \cong f\left(K_{2}\right)$ in $S^{3}$.

Proof. Let us consider a 3-components Brunnian link $L=k \cup L_{1} \cup L_{2}$ depicted in Figure 8.

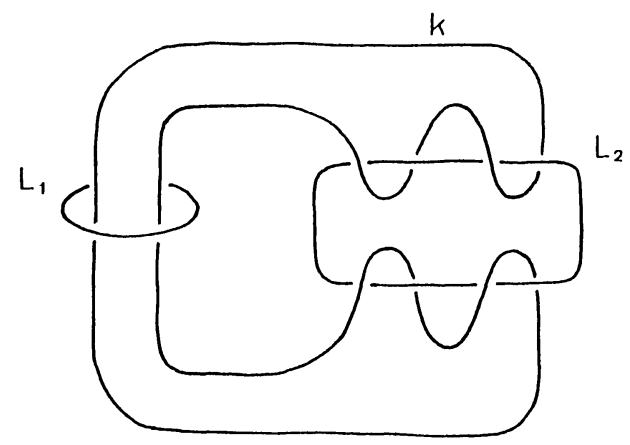

Figure 8

We denote the meridian-longitude pair of $L_{i}$ by $\left(m_{i}, l_{i}\right) \quad(i=$ $1,2)$. Let $t$ be a knot ambient isotopic to $f(C)$, where $C$ is a core of $V$, and $(m, l)$ a meridian-longitude pair of $t$. To obtain the required pattern, remove a tubular neighborhood $N\left(L_{i}\right)$ and glue the knot exterior $E(t)=S^{3}-$ int $N(t)$ so that $m_{i}=l$ and $l_{i}=m$. Then, for $i=1,2$, the result $\left(S^{3}-\right.$ int $\left.N\left(L_{i}\right)\right) \cup_{\substack{m_{l}=l \\ l_{l}=m}}\left(S^{3}-\right.$ int $\left.N(t)\right)$ is again $S^{3}$, and we have new knots $K_{3-i}$ and $\widetilde{L}_{3-i}$ as the imaḡes of $k$ and $L_{3-i}$ respectively. It is easy to see that both $K_{3-i}$ and $\widetilde{L}_{3-i}$ are unknotted in $S^{3}$. Thus the exterior $V$ of $\widetilde{L}_{3-i}$ containing $K_{3-i}$ forms a pattern $\left(V, K_{3-i}\right)$. In this way we get two patterns $\left(V, K_{1}\right)$ and $\left(V, K_{2}\right)$. By the construction, for the faithful 
embedding $f: V \hookrightarrow S^{3}, f\left(K_{1}\right) \cong f\left(K_{2}\right)$ does hold in $S^{3}$. In fact, $f\left(K_{1}\right) \cong f\left(K_{2}\right)$ can be described as the knot obtained from $k$ in Figure 8 by simultaneously replacing a neighborhood of a meridian disk of each of $L_{1}$ and $L_{2}$ by a tube knotted according to the given knot $t$.

From now on we prove $\left(V, K_{1}\right) \nsim\left(V, K_{2}\right)$ by showing $\operatorname{wrap}_{V}\left(K_{1}\right)$ $\neq \operatorname{wrap}_{V}\left(K_{2}\right)$. Clearly $\operatorname{wrap}_{V}\left(K_{1}\right) \leq 2$ and $\operatorname{wrap}_{V}\left(K_{2}\right) \leq 4$. Since wind $_{V}\left(K_{2}\right)=0, \operatorname{wrap}_{V}\left(K_{2}\right)$ must be even. Now we assume $\operatorname{wrap}_{V}\left(K_{2}\right)$ $=2$. Then there exists a disk $D_{2}$ in

$$
V=\left(S^{3}-\operatorname{int} N\left(L_{1}\right)\right) \cup_{\substack{m_{1}=l \\ l_{1}=m}}\left(S^{3}-\operatorname{int} N(t)\right)-\operatorname{int} N\left(L_{2}\right)
$$

such that $D_{2} \cap K_{2}=D_{2} \cap k$ consists of two points and $\partial D_{2}=l_{2}$. Extending $D_{2}$, we may assume $\partial D_{2}=L_{2}$. Let $D_{k}$ be the disk depicted in Figure $9(1)$, such that $\partial D_{k}=k$. We remark that $D_{k} \cap L_{2}$ consists of four points $p_{1}, p_{2}, q_{1}, q_{2}$ (see Figure $9(1)$ ).

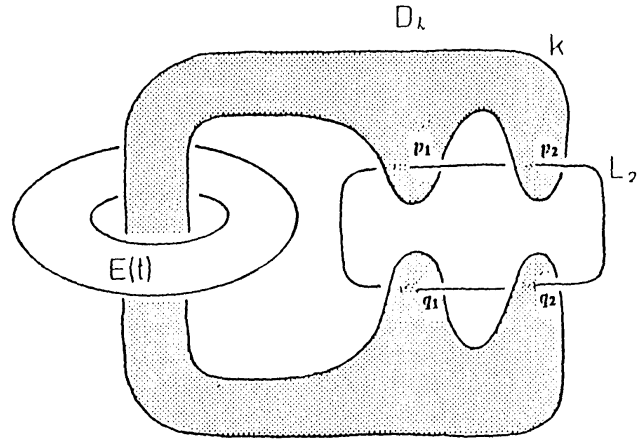

(1)

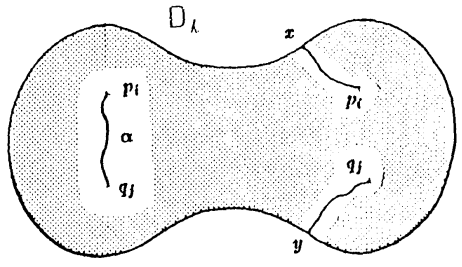

(2)

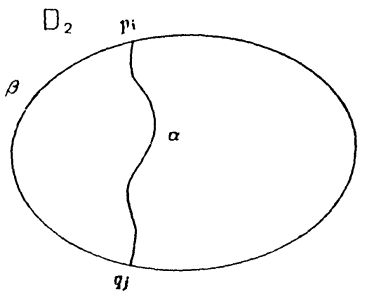

(3)

FIGURE 9

From the assumption we see that the boundary of arc components of $D_{2} \cap D_{k}$ in $D_{k}$ consists of six points $p_{1}, p_{2}, q_{1}, q_{2}, x, y$ (see Figure $9(2))$. Considering the orientations, there exists an arc component $\alpha$ of $D_{2} \cap D_{k}$ joining $p_{i}$ and $q_{j}$ for some $i, j$ (see Figure 9 (2)(3)). Let $\beta$ be an arc of $L_{2}$ connecting $p_{i}$ and $q_{j}$, and $D$ a disk in $D_{2}$ bounded by $\alpha \cup \beta$. Then $\alpha \cup \beta$ clearly has winding number one in the solid torus $S^{3}-$ int $N\left(L_{1}\right)$, which is knotted as $N(t)$ in $S^{3} \supset V$. 
This contradicts, via Lemma 2.1, that $\alpha \cup \beta=\partial D$. Hence we can conclude $\operatorname{wrap}_{V}\left(K_{2}\right) \neq 2$, and applying the same argument we get also $\operatorname{wrap}_{V}\left(K_{2}\right) \neq 0$. It follows that $\operatorname{wrap}_{V}\left(K_{2}\right)=4$. We see $\operatorname{wrap}_{V}\left(K_{1}\right)=$ 2 easily as follows. If $\operatorname{wrap}_{V}\left(K_{1}\right) \neq 2$, then $\operatorname{wrap}_{V}\left(K_{1}\right)=0$. However this means $\operatorname{wrap}_{V}\left(K_{2}\right)=0$, thus $\operatorname{wrap}_{V}\left(K_{1}\right)=2$.

In this way we get the required patterns.

This result can be generalized to

Corollary 3.4. For any knot $K$ in $S^{3}$ and any faithful embedding $f$ from $V$ into $S^{3}$, there exist patterns $\left(V, K_{1}\right)$ and $\left(V, K_{2}\right)$ such that $K_{i} \cong K$ in $S^{3}(i=1,2)$, which satisfy the following properties:

(1) $\left(V, K_{1}\right) \nsim\left(V, K_{2}\right)$.

(2) $f\left(K_{1}\right) \cong f\left(K_{2}\right)$ in $S^{3}$ for the embedding $f$ from $V$ into $S^{3}$.

Proof. Let $\left(V, k_{1}\right)$ and $\left(V, k_{2}\right)$ be the patterns constructed in Theorem 3.3 depending upon the embedding $f$. Since $k_{i}(i=1,2)$ is trivial in $S^{3}$, we can locally replace an unknotted arc of $k_{i}$ by a knotted arc (with a suitable direction) so that the resulting knot $K_{i}$ represents $K$ in $S^{3}$. Then it follows from the choice of $\left(V, k_{1}\right)$ and $\left(V, k_{2}\right)$ that $\left(V, K_{1}\right)$ and $\left(V, K_{2}\right)$ are the required patterns.

Acknowledgments. I express my gratitude to Professor Tetsuo Shibuya for helpful communications and for giving an example in Remark 1.2 (1). The construction of Theorem 3.3 is due to Professor Makoto Sakuma, I want also to thank him. Finally I wish to thank the referee for useful comments.

\section{REFERENCES}

[1] G. Burde and H. Zieschang, Knots, de Gruyter Studies in Math., vol. 5, Walter de Gruyter, 1985.

[2] J. Hempel, 3-manifolds, Ann. of Math. Stud., vol. 86, Princeton Univ. Press, Princeton, NJ, 1976.

[3] W. Jaco, Lectures on the three manifold topology, CBMS Regional Conf. Ser. in Math., No. 43, Amer. Math. Soc., Providence, RI, 1980.

[4] W. Jaco and P. Shalen, Seifert fibered spaces in 3-manifolds, Mem. Amer. Math. Soc., 220 (1979).

[5] K. Johannson, Homotopy equivalences of 3-manifolds with boundaries, Lecture Notes in Math., vol. 761, Springer-Verlag, Berlin and New York, 1979.

[6] J. Morgan and H. Bass, The Smith Conjecture, Pure Appl. Math., Academic Press, San Diego, CA, 1984.

[7] D. Rolfsen, Knots and links, Mathematics Lecture Series, No. 7, Publish or Perish, Berkeley, CA, 1976. 
[8] H. Schubert, Knoten und Vollringe, Acta Math., 90 (1953), 131-286.

[9] H. Seifert, On the homology invariants of knots, Quart. J. Math. Oxford Ser. (2), 1 (1950), 23-32.

[10] T. Soma, The Gromov invariant of links, Invent. Math., 64 (1981), 445-454.

Received July 24, 1991 and in revised form September 7, 1992. This research was supported in part by Grant-in-Aid for Encouragement of Young Scientists 03740047, The Ministry of Education, Science and Culture.

DepartMent OF MATHEMATICS

College of Humanities \& Sciences

NIHON UNIVERSITY

Sakurajosui 3-25-40, Setagaya-ku

TOKYO 156, JAPAN 



\title{
PACIFIC JOURNAL OF MATHEMATICS
}

Founded by

\author{
E. F. BECKenBaCH (1906-1982) F. WolF (1904-1989)
}

\section{EDITORS}

Sun-Yung A. Chang

(Managing Editor)

University of California

Los Angeles, CA 90024-1555

chang@math.ucla.edu

F. Michael Christ

University of California

Los Angeles, CA 90024-1555

christ@math.ucla.edu

Herbert Clemens

University of Utah

Salt Lake City, UT 84112

clemens@math.utah.edu

\author{
R. FInN
}

THOMAS ENRIGHT

University of California, San Diego

La Jolla, CA 92093

tenright@ucsd.edu

Nicholas ERColani

University of Arizona

Tucson, AZ 85721

ercolani@math.arizona.edu

Stanford University

Stanford, CA 94305

finn@gauss.stanford.edu

VAUghan F. R. JoNES

University of California

Berkeley, CA 94720

vfr@math.berkeley.edu

\section{SUPPORTING INSTITUTIONS}

STEVEN KeRCKHOFF

Stanford University

Stanford, CA 94305

spk@gauss.stanford.edu

MARTIN ScharLemanN

University of California

Santa Barbara, CA 93106

mgscharl@henri.ucsb.edu

HAROLd STARK

University of California, San Diego La Jolla, CA 92093

V. S. VARADARAJAN

University of California

Los Angeles, CA 90024-1555

vsv@math.ucla.edu

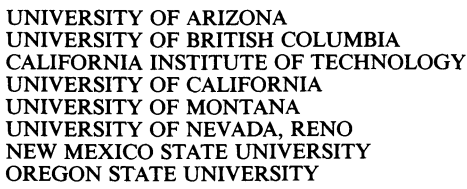

The Supporting Institutions listed above contribute to the cost of publication of this Journal, but they are not owners or publishers and have no responsibility for its content or policies.

Mathematical papers intended for publication in the Pacific Journal of Mathematics should be in typed form or offset-reproduced (not dittoed), double spaced with large margins. Please do not use built up fractions in the text of the manuscript. However, you may use them in the displayed equations. Underline Greek letters in red, German in green, and script in blue. The first paragraph must be capable of being used separately as a synopsis of the entire paper. In particular it should contain no bibliographic references. Please propose a heading for the odd numbered pages of less than 35 characters. Manuscripts, in triplicate, may be sent to any one of the editors. Please classify according to the 1991 Mathematics Subject Classification scheme which can be found in the December index volumes of Mathematical Reviews. Supply name and address of author to whom proofs should be sent. All other communications should be addressed to the managing editor, or Julie Honig, University of California, Los Angeles, California 90024-1555.

There are page-charges associated with articles appearing in the Pacific Journal of Mathematics. These charges are expected to be paid by the author's University, Government Agency or Company. If the author or authors do not have access to such Institutional support these charges are waived. Single authors will receive 75 free reprints; joint authors will receive a total of 100 free reprints. Additional copies may be obtained at cost in multiples of 50 .

The Pacific Journal of Mathematics (ISSN 0030-8730) is published monthly except for July and August. Regular subscription rate: $\$ 200.00$ a year (10 issues). Special rate: $\$ 100.00$ a year to individual members of supporting institutions.

Subscriptions, orders for numbers issued in the last three calendar years, and changes of address should be sent to Pacific Journal of Mathematics, P.O. Box 969, Carmel Valley, CA 93924, U.S.A. Old back numbers obtainable from Kraus Periodicals Co., Route 100, Millwood, NY 10546.

The Pacific Journal of Mathematics at P.O. Box 969, Carmel Valley, CA 93924 (ISSN 0030-8730) is published monthly except for July and August. Second-class postage paid at Carmel Valley, California 93924, and additional mailing offices. Postmaster: send address changes to Pacific Journal of Mathematics, P.O. Box 969, Carmel Valley, CA 93924.

PUBLISHED BY PACIFIC JOURNAL OF MATHEMATICS, A NON-PROFIT CORPORATION

This publication was typeset using $\mathcal{A} \mathcal{M S}-\mathrm{T}_{\mathrm{E}} \mathrm{X}$, the American Mathematical Society's $\mathrm{T}_{\mathrm{E}} \mathrm{X}$ macro system. Copyright (c) 1993 by Pacific Journal of Mathematics 


\section{PACIFIC JOURNAL OF MATHEMATICS}

Volume $161 \quad$ No. $2 \quad$ December 1993

On the method of constructing irreducible finite index subfactors of 201 Popa

FLORIN PETRE BOCA

Brownian motion and the heat semigroup on the path space of a

233 compact Lie group

JAY BARRY EPPERSON and TERRY M. LOHRENZ

Horizontal path spaces and Carnot-Carathéodory metrics

255

ZHONG GE

Biholomorphic convex mappings of ball in $\mathbb{C}^{n}$

SHENG GONG, SHI KUN WANG and QI HUANG YU

The Temperley-Lieb algebra at roots of unity

FREDERICK MiCHAEL GOODMAN and HANS WENZL

Jordan analogs of the Burnside and Jacobson density theorems

LUZIUS GRÜNENFELDER, M. OLMLADIČ and HEYDAR RADJAVI

Elliptic representations for $\mathrm{Sp}(2 n)$ and $\mathrm{SO}(n)$

REBECCA A. Herb

Reflexivity of subnormal operators

JOHN MCCARTHY

Knotting trivial knots and resulting knot types

KiMiHiko MOTEGI

Commutativity of selfadjoint operators

MitsURU UCHIYAMA

Correction to: "One-dimensional Nash groups" 